\title{
Tensor models for black hole probes
}

\section{Nick Halmagyi and Swapnamay Mondal}

Sorbonne Université, CNRS, Laboratoire de Physique Théorique et hautes Énergies, LPTHE, F-75005 Paris, France

CNRS, UMR 7589, LPTHE, 75005, Paris, France

E-mail: halmagyi@lpthe.jussieu.fr, swapno@lpthe.jussieu.fr

ABstract: The infrared dynamics of the SYK model, as well as its associated tensor models, exhibit some of the non trivial features expected of a holographic dual of near extremal black holes. These include developing certain symmetries of the near horizon geometry and exhibiting maximal chaos. In this paper we present a generalization of these tensor models to include fields with fewer tensor indices and which can be thought of as describing probes in a black hole background. In large $N$ limit, dynamics of the original model remain unaffected by the probe fields and some of the four point functions involving probe fields exhibit maximal chaos, a non trivial feature expected of a black hole probe.

KEywords: 1/N Expansion, Black Holes in String Theory, Conformal Field Theory, AdSCFT Correspondence

ARXIV EPRINT: 1711.04385 


\section{Contents}

1 Introduction 1

2 Interactions in the $\mathrm{D}=3$ uncolored model 2

2.1 A lightening review of the KT model 2

2.2 A class of solvable models 5

3 Uncolored probe model $\quad 6$

3.1 Propagators

3.2 Four point functions 8

$3.2 .1\langle\psi \psi \psi \psi\rangle \quad{ }^{3.2} \quad 8$

$\begin{array}{llr}3.2 .2\langle\kappa \kappa \kappa \kappa\rangle & 9\end{array}$

$\begin{array}{lll}3.2 .3\langle\psi \psi \kappa \kappa\rangle & 10\end{array}$

$\begin{array}{lll}3.3 \text { Chaos } & 11\end{array}$

3.4 Spectrum 12

$\begin{array}{ll}\text { 3.5 Adding additional fields } & 14\end{array}$

4 Colored probe model $\quad 16$

$\begin{array}{lll}5 & \text { Discussion and future directions } & 17\end{array}$

$\begin{array}{ll}\text { A Four point functions with maximal chaos } & 17\end{array}$

\section{Introduction}

The study of quantum mechanical models dual to gravitational systems in two dimensions remains a fascinating and difficult arena of research. Quite notably, simple and solvable examples of this duality have proved to be difficult to construct. Somewhat recently however, the fermionic quantum mechanics of SYK model [1] has been proposed as a system holographically dual to gravity and this has been studied extensively [2-16]. A key motivating factor for proposing the SYK model as a holographic dual of a black hole background is the fact that the out of time order four-point correlation functions saturate the so-called maximal chaos bound [17] which has been shown to hold in the bulk. Another important feature of the SYK model is that the emergent conformal symmetry is both spontaneously and explicitly broken, which suggests that it is dual to a near AdS2 background.

In an interesting development, it has been shown [18] that to leading order in the large $N$ expansion, the SYK model (which is disordered, hence not fully quantum mechanical) is identical to the fermionic tensor model of $[19,20]$. This has been subsequently generalized in a number of interesting directions [21-27].

In this paper we couple the Klebanov-Tarnopolsky model [21] and Gurau-Witten model [18] to lower-index fields and interpret the resulting quantum mechanical models 


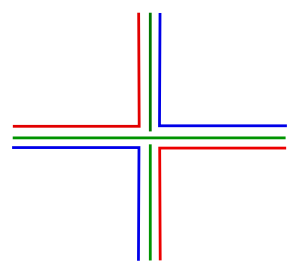

Figure 1. Diagrammatic representation of vertices of KT model: colored lines represent resolved indices. Red, green and blue lines represent respectively first, second and third indices of a the field $\psi_{a b c}$.

as holographically dual to probes in a black hole background. This is in part motivated by examples where matrix models have been coupled to vector matter, where the latter can be considered as probes in a background described by the former. ${ }^{1}$ The models we study are obtained by adding interactions between the original tensors of $[18,21]$ and tensors of lower rank. ${ }^{2}$ To leading order in the $1 / N$ expansion, the additional tensors do not affect the physics of original tensors, thus they can rightfully be thought of as probes. Furthermore, we find that a host of four point functions exhibit maximal chaos ${ }^{3}$ and it is this feature which qualifies these models as toy models for probes in a black hole background.

The rest of the paper is organized as follows. In section 2, we give brief introduction to the Klebanov-Tarnopolsky model [21] and discuss a class of possible modifications, that remain solvable in large $N$ limit and in deep infrared. In section 3 , we consider simplest such model and discuss the propagators, four point functions, primaries and Lyapunov coefficients. We also comment on possible further modifications of this model, that retain the necessary physics. In section 4, we discuss similar modifications of Gurau-Witten model [18]. Finally in section 5 we discuss future directions.

\section{Interactions in the $\mathrm{D}=3$ uncolored model}

\subsection{A lightening review of the KT model}

The KT model [21] contains a single real fermionic tensor $\psi_{a b c}$ of rank 3. Each index transforms as a vector under $\mathrm{SO}(N)$. To differentiate the three copies of $\mathrm{SO}(N)$ we write the first as $\mathrm{SO}(N)_{1}$, the second as $\mathrm{SO}(N)_{2}$ and the third as $\mathrm{SO}(N)_{3}$. The Hamiltonian is taken to be

$$
H=\frac{g_{0}}{4 N^{3 / 2}} \psi_{a_{1} b_{1} c_{1}} \psi_{a_{1} b_{2} c_{2}} \psi_{a_{2} b_{1} c_{2}} \psi_{a_{2} b_{2} c_{1}}
$$

whose diagrammatic representation is given in figure 1 . This model can be obtained by "uncoloring" [32] the $\mathrm{D}=3$ Gurau-Witten model [18]. The large $N$ limit $[19,20]$ is defined as taking $N \rightarrow \infty$ while keeping $g_{0}$ fixed. In this limit it is the melonic grpahs which

\footnotetext{
${ }^{1}$ See $[28,29]$ for an interesting recent example relevant for black hole physics, although this model is not maximally chaotic [30].

${ }^{2} \mathrm{~A}$ model which couples rank three and rank one tensor fields has been considered in [26] but in our model we preserve the interactions purely between the three-index fields ( $D$ index fields for color $D$ ).

${ }^{3}$ Recently [31] presented another example of probes exhibiting maximal chaos.
} 


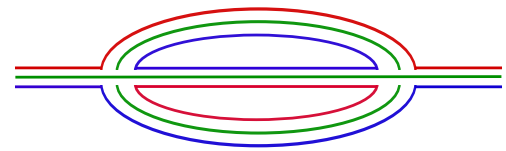

Figure 2. simplest melonic correction to the propagator.

contribute to leading order in $1 / N$ and the simplest correction to the propagator comes from the melonic graph in figure 2. A factor of $N^{3}$, coming from fields propagating in loops, cancels the $1 / N^{3}$ coming from the two vertices, giving an overall factor of $N^{0}$. Additional melonic corrections to the propagator of the same order are obtained by replacing any of the internal or external propagators in the diagram by this melonic diagram itself. In the large $N$ limit this class of diagrams ${ }^{4}$ constitute the complete leading corrections to the free propagator. They can be summed up to give the exact propagator in deep IR as follows. Denoting $G\left(t_{1}, t_{2}\right)$ to be the propagator and $\Sigma\left(t_{1}, t_{2}\right)$ to be the 1PI two point function to leading order in $1 / N$, one has

$$
\Sigma\left(t_{1}, t_{2}\right)=g_{0}^{2} G\left(t_{1}, t_{2}\right)^{3}
$$

By definition

$$
G(\omega)=\frac{1}{-i \omega-\Sigma(\omega)},
$$

and in the deep infrared, $i \omega$ can be ignored. In position space, one obtains

$$
\int d t G\left(t_{1}, t\right) \Sigma\left(t, t_{2}\right)=-\delta\left(t_{1}-t_{2}\right)
$$

giving the following Schwinger-Dyson equation

$$
g_{0}^{2} \int d t G\left(t_{1}, t\right) G\left(t, t_{2}\right)^{3}=-\delta\left(t_{1}-t_{2}\right),
$$

in deep IR. The result (2.5) is invariant under the conformal transformations ${ }^{5} t \rightarrow f(t)$

$$
\begin{aligned}
& G\left(t_{1}, t_{2}\right) \rightarrow\left|\frac{d f\left(t_{1}\right)}{d t_{1}} \frac{d f\left(t_{2}\right)}{d t_{2}}\right|^{1 / 4} G\left(f\left(t_{1}\right), f\left(t_{2}\right)\right), \\
& \Sigma\left(t_{1}, t_{2}\right) \rightarrow\left|\frac{d f\left(t_{1}\right)}{d t_{1}} \frac{d f\left(t_{2}\right)}{d t_{2}}\right|^{3 / 4} \Sigma\left(f\left(t_{1}\right), f\left(t_{2}\right)\right) .
\end{aligned}
$$

Thus the system develops an emergent conformal symmetry in the deep IR. The solution to $(2.5)$ is

$$
G_{c}(t)=\frac{b}{|t|^{1 / 2}} \operatorname{sgn}(t), \quad \text { where, } \quad b^{4}=\frac{1}{4 \pi g_{0}^{2}},
$$

which spontaneously breaks the conformal symmetry to $\mathrm{SL}(2, \mathbb{R})$.

\footnotetext{
${ }^{4}$ Joining the ends of a propagator gives a vacuum diagram. Thus this class of diagrams also give leading contributions to free energy in large $N$ limit. Joining the ends turns the external lines into internal ones and thus one gets an extra factor of $N^{3}$. Since the propagators were $\mathcal{O}\left(N^{0}\right)$, this means that free energy scales as $N^{3}$, which is good since the number of fields scales as $N^{3}$.

${ }^{5}$ In one dimension conformal group contains all reparameterizations.
} 


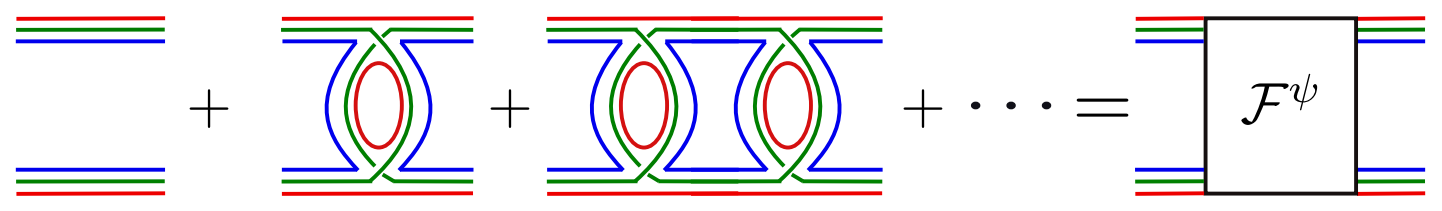

Figure 3. Leading contributions to "gauge invariant four point function" in large $N$ limit.

Next one considers the "gauge invariant" four point function, which has the following structure

$$
\begin{aligned}
& \frac{1}{N^{6}} \sum_{\substack{a_{1}, b_{1}, c_{1}, a_{2}, b_{2}, c_{2}}}\left\langle\psi_{a_{1} b_{1} c_{1}}\left(t_{1}\right) \psi_{a_{1} b_{1} c_{1}}\left(t_{2}\right) \psi_{a_{2} b_{2} c_{2}}\left(t_{3}\right) \psi_{a_{2} b_{2} c_{2}}\left(t_{4}\right)\right\rangle \\
= & G\left(t_{1}, t_{2}\right) G\left(t_{3}, t_{4}\right)+\frac{1}{N^{3}} \mathcal{F}\left(t_{1}, t_{2} ; t_{3}, t_{4}\right),
\end{aligned}
$$

where $\mathcal{F}$ is given by the sum of ladder diagrams shown in figure 3. A ladder with $n$ rungs is denoted as $\mathcal{F}_{n}$ and can be obtained from $\mathcal{F}_{n-1}$ by acting with the kernel $K$ :

$$
\begin{aligned}
\mathcal{F}_{n}\left(t_{1}, t_{2} ; t_{3}, t_{4}\right) & =\int d t d t^{\prime} K\left(t_{1}, t_{2} ; t, t^{\prime}\right) \mathcal{F}_{n-1}\left(t, t^{\prime} ; t_{3}, t_{4}\right), \\
\text { where } \quad K\left(t_{1}, t_{2} ; t_{3}, t_{4}\right) & =-3 g_{0}^{2} G\left(t_{1}, t_{3}\right) G\left(t_{2}, t_{4}\right) G\left(t_{3}, t_{4}\right)^{2} .
\end{aligned}
$$

The factor of 3 stems from the fact that in figure 3 red lines are in a somewhat different status. There are other two class of diagrams where green and blue lines have the special status respectively. The kernel $K$ commutes with $\mathrm{SL}(2, \mathbb{R})$ generators. Given any generator $J$ of $\operatorname{SL}(2, \mathbb{R})$, one has

$$
\left(J_{1}+J_{2}\right) K\left(t_{1}, t_{2} ; t_{3}, t_{4}\right)=K\left(t_{1}, t_{2} ; t_{3}, t_{4}\right)\left(J_{3}+J_{4}\right) .
$$

Here $J_{i}$ acts on time $t_{i}$.

Eigenfunctions and eigenvalues of $K$ can be labelled by those of $\operatorname{SL}(2, \mathbb{R})$ Casimir. They appear in a discreet and a continuum series. The discreet one is labelled by $h=2 n$ and the continuum one is labelled by $h=1 / 2+i s$. Corresponding eigenvalues of $K$ are given by $[2]$

$$
k_{c}(h)=-\frac{3}{2} \frac{\tan \left(\frac{\pi h}{2}-\frac{\pi}{4}\right)}{h-1 / 2}
$$

Using (2.9) one can sum up the ladder diagrams to obtain

$$
\mathcal{F}=\left(1+K+K^{2}+\ldots\right) \mathcal{F}_{0}=\frac{1}{1-K} \mathcal{F}_{0} .
$$

Combining (2.10) with the fact that

$$
\mathcal{F}_{0}\left(t_{1}, t_{2} ; t_{3}, t_{4}\right) \equiv-G\left(t_{1}, t_{3}\right) G\left(t_{2}, t_{4}\right)+G\left(t_{1}, t_{4}\right) G\left(t_{2}, t_{3}\right)
$$

preserves the $\operatorname{SL}(2, \mathbb{R})$ symmetry, we see that one can use $\operatorname{SL}(2, \mathbb{R})$ symmetry to evaluate (2.12), although the $K=1$ subspace requires special care and ultimately results in the breaking of the conformal symmetry. 

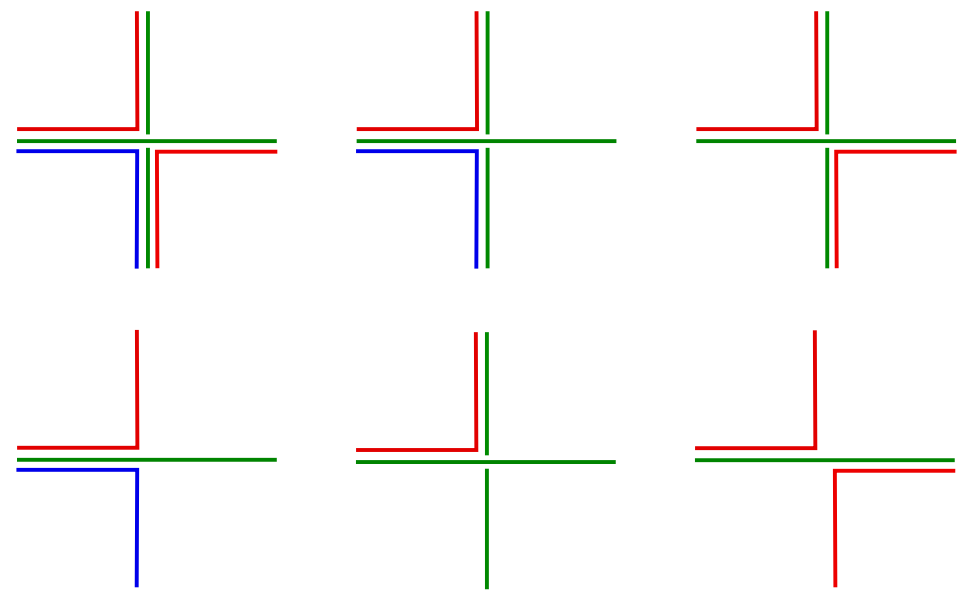

Figure 4. various interaction obtained by removing resolved lines from KT model: red, green and blue resolved lines denote first, second and third resolved indices respectively. E.g. the field $\kappa^{(12)}$ will have one red and one green lines, the field $\eta^{(3)}$ will have one blue line and so on. Permuting colors will give other vertices of same class.

\subsection{A class of solvable models}

The KT model can be thought of as a toy model for near extremal black holes. A model which includes probes of this black hole should have additional fields which preserve the property of "maximal chaos" and we will present such a model later in section 3 . In this section we investigate some modifications of KT model, which remain solvable at large $N$.

We begin with the observation that removing open lines from figure 2 does not change the $N$ dependence. For example removing the open blue line from figure 2 gives the second diagram of figure 6 . This can be thought of as a correction to the propagator for a field carrying two indices (represented by green and red lines) coming from an interaction vertex (see last vertex of figure 5) that can be obtained from figure 1 by removing one blue line. This suggests that adding fields carrying less number of indices and interacting through such an interaction leads to new theories with large $\mathrm{N}$ structure similar to that of the KT model and therefore also to the SYK model.

A non exhaustive list of such interaction vertices, obtained from 1 by removing various open lines is given in figure 4 . Some of the vertices shown in figure 4 have previously been considered in [33]. Also see [34]. These interactions include the following new fields:

$$
\kappa_{a b}^{(12)}, \kappa_{b c}^{(23)}, \kappa_{a c}^{(13)}, \eta_{a}^{(1)}, \eta_{b}^{(2)}, \eta_{c}^{(3)} .
$$

Here $\kappa^{(i j)}$ carries the $i^{\text {th }}$ and $j^{\text {th }}$ indices of $\psi$, whereas $\eta^{(i)}$ carries only $i^{\text {th }}$ index of $\psi$. This implies that $\kappa^{(i j)}$ transforms as bifundamental under $\mathrm{SO}(N)_{i} \times \mathrm{SO}(N)_{j}$ and trivially under the remaining $\mathrm{SO}(N)$ while $\eta^{(i)}$ transforms as fundamental of $\mathrm{SO}(N)_{i}$ and trivially under remaining two $\mathrm{SO}(N)$ symmetries.

Among all the interactions of the above kind, only a few are relevant in determining the large $N$ physics. In figure 2 , if one removes any loops, the diagram becomes subleading in $1 / N$. We claim that only 2 such vertices (up to color permutation) as shown in 

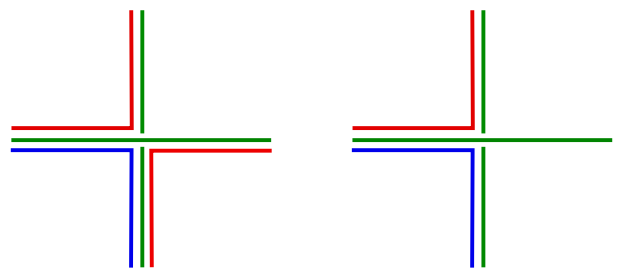

Figure 5. vertices (up to color permutations) relevant for the large $N$ structure.
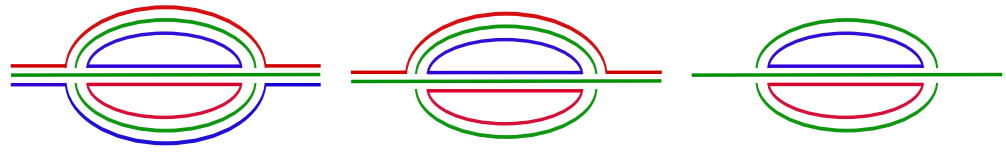

Figure 6. Leading melonic contributions to various propagators.

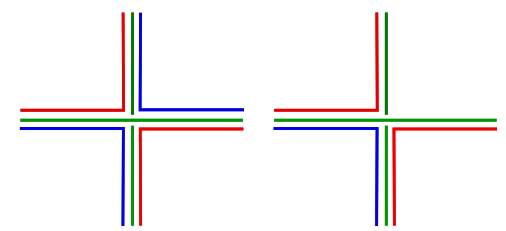

Figure 7. Diagrammatic representation of all the vertices appearing in (3.1).

figure 5 contribute to the leading order graphs. These interactions, along with original interaction (2.1) give the new leading contributions to various propagators, as shown in figure 6 .

\section{Uncolored probe model}

In this section we present a class of models motivated by our discussion in section 2.2 and compute the four point functions and Lyapunov coefficient. The Hamiltonian for the simplest of our models is given by

$$
H=\frac{g_{0}}{4 N^{3 / 2}} \psi_{a_{1} b_{1} c_{1}} \psi_{a_{1} b_{2} c_{2}} \psi_{a_{2} b_{1} c_{2}} \psi_{a_{2} b_{2} c_{1}}+\frac{g_{1}}{2 N^{3 / 2}} \psi_{a_{1} b_{1} c_{1}} \psi_{a_{2} b_{2} c_{1}} \kappa_{a_{1} b_{2}} \kappa_{a_{2} b_{1}}
$$

where we have introduced factors of $N$ in the interactions such that both $g_{0}$ and $g_{1}$ are $\mathcal{O}\left(N^{0}\right)$. Diagrammatic representations of the interaction vertices of (3.1) are given in figure 7. We refer to the first term as $\mathrm{KT}$ term, which can be thought of as describing dynamics of a black hole, whose effective degrees of freedoms are captured in the field $\psi$. We refer to $\kappa$ (which is same as $\kappa^{(12)}$ in last section) as the "probe field" and to the second term of (3.1) as the "probe term", which is to be thought of as describing the interaction between a black hole and the probe. ${ }^{6}$ Interactions involving only probe fields are subleading and not considered in this work.

\footnotetext{
${ }^{6} \mathrm{KT}$ model has some extra Goldstone modes [24] compared to SYK model which are unaffected by the probe term.
} 


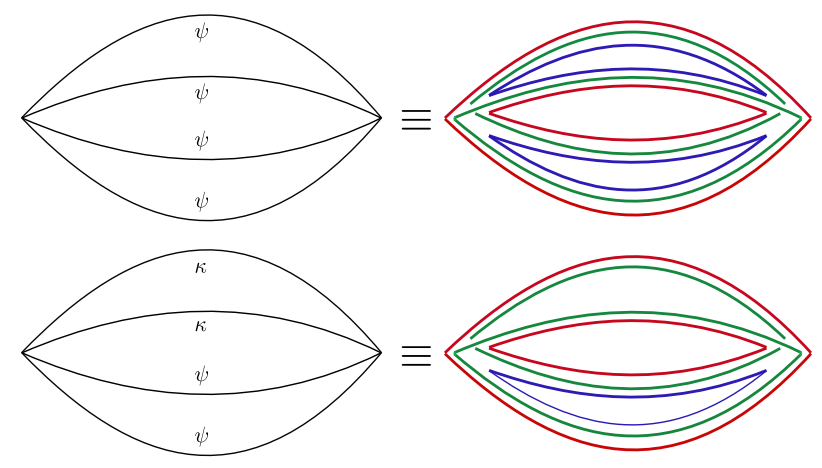

Figure 8. Simplest vacuum diagrams for (3.1).

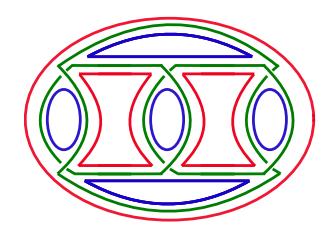

Figure 9. A subleading bubble in KT theory of order $N^{2}$.

The simplest vacuum diagrams for (3.1) are shown in figure 8. The upper diagram, coming from KT term, scales as $N^{3}$, whereas the lower diagram, coming from the probe term, scales as $N^{2}$ and thus gives subleading contributions to free energy. This implies that in the large $N$ limit, thermodynamic properties of the system is entirely determined by the KT term. There are also subleading diagrams arising purely from KT interactions as well. For example the diagram in figure 9 also scales as $N^{2}$.

\subsection{Propagators}

$G^{\psi}$. Propagators for fields $\psi$ is unaffected by the new vertex in the large $N$ limit. This is because the contribution to $G^{\psi}$ from the probe interaction comes from cutting a $\psi$ line in the lower melon of figure 8 . But this scales as $1 / N$ and hence suppressed. Thus in large $N$ limit and in deep infra red, (2.7) continues to hold. From now on we will refer to $G^{\psi}$ simply by $G$.

$G^{\kappa}$. Simplest contribution to $G^{\kappa}$ comes from cutting a $\kappa$ line in the lower melon of figure 8 . This removes two loops and the resultant diagram scales as $\mathcal{O}\left(N^{0}\right)$. Additionally, one can replace an internal $\kappa$ line with such a melon and still get a correction of order $\mathcal{O}\left(N^{0}\right)$. One can continue doing this and following logic similar to $\psi$ propagators, one gets the following Schwinger Dyson equation for $G^{\kappa}$

$$
g_{1}^{2} \int d t G^{\kappa}\left(t_{1}, t\right)\left(G^{\psi}\left(t, t_{2}\right)\right)^{2} G^{\kappa}\left(t, t_{2}\right)=-\delta\left(t_{1}-t_{2}\right),
$$

whose solution is

$$
G^{\kappa}\left(t_{1}, t_{2}\right)=\frac{g_{0}}{g_{1}} G^{\psi}\left(t_{1}, t_{2}\right)=\frac{b g_{0}}{g_{1}} \frac{\operatorname{sgn}\left(t_{1}-t_{2}\right)}{\left|t_{1}-t_{2}\right|^{1 / 2}} .
$$


We emphasize that $G^{\kappa}$ is of same order as $G^{\psi}$ in the $1 / N$ expansion but not in a $g_{0}$ expansion. ${ }^{7}$ At the level of propagators there is an emergent conformal symmetry which is spontaneously broken to $\mathrm{SL}(2, \mathbb{R})$.

\subsection{Four point functions}

We now consider four point functions involving various combinations of $\psi$ and $\kappa$ fields. All connected graphs which contribute to these four point functions can be obtained by cutting two lines from appropriate melon diagrams.

\subsection{1 $\langle\psi \psi \psi \psi \psi\rangle$}

Like the propagator, four point functions of $\psi$ fields are not affected by the presence of new interactions to leading order. Therefore the leading contribution to $\mathcal{F}$, the connected part of $\frac{1}{N^{6}}\left\langle\psi_{a_{1} b_{1} c_{1}}\left(t_{1}\right) \psi_{a_{1} b_{1} c_{1}}\left(t_{2}\right) \psi_{a_{2} b_{2} c_{2}}\left(t_{3}\right) \psi_{a_{2} b_{2} c_{2}}\left(t_{4}\right)\right\rangle$, comes from summing up the ladder diagrams shown in figure 3 and is given by $(2.12)$, which we repeat for convenience ${ }^{8}$

$$
\mathcal{F}=\left(1+K+K^{2}+\ldots\right) \mathcal{F}_{0}=\frac{1}{1-K} \mathcal{F}_{0}
$$

where, $K\left(t_{1}, t_{2} ; t_{3}, t_{4}\right)=-3 g_{0}^{2} G\left(t_{1}, t_{3}\right) G\left(t_{2}, t_{4}\right) G\left(t_{3}, t_{4}\right)^{2}$,

$$
\mathcal{F}_{0}\left(t_{1}, t_{2} ; t_{3}, t_{4}\right)=-G\left(t_{1}, t_{3}\right) G\left(t_{2}, t_{4}\right)+G\left(t_{1}, t_{4}\right) G\left(t_{2}, t_{3}\right) .
$$

Following [2] we define in the conformal limit, the normalized four point function:

$$
\frac{\mathcal{F}\left(t_{1}, t_{2} ; t_{3}, t_{4}\right)}{G\left(t_{12}\right) G\left(t_{34}\right)}=\mathcal{F}(\chi), \quad \text { where } \quad \chi=\frac{t_{12} t_{34}}{t_{13} t_{24}},
$$

and note that $\mathcal{F}(\chi)$ was evaluated in [2].

A central point of [2] is that in the strict conformal limit, the four point function of the SYK-model diverges. In addition to the finite and conformally invariant component of the four point function $\mathcal{F}(\chi)$, there is an additional non-trivial component $\mathcal{F}\left(t_{1}, t_{2} ; t_{3}, t_{4}\right)$ which diverges and breaks conformal symmetry (thus cannot be expressed as a function of $\chi$ alone). This non-conformal component of $\mathcal{F}^{\psi}$ is denoted $\mathcal{F}_{h=2}^{\psi}$ due to the manner in which the eigenvalues of $K$ are parameterized in [2]. The unit eigen-subspace of $K$ corresponds to $h=2$ and from (3.4) we see that it is this subspace which leads to the divergence. One proceeds by regulating the spectrum of $K$ and the first non-trivial correction to the unit eigenvalue of $K$ is of order $\frac{1}{\beta g_{0}}$, which in turn gives a contribution of order

$$
\mathcal{F}_{h=2}^{\psi} \sim \beta g_{0}+\ldots
$$

where the ellipsis represent lower order terms in the expansion in $\beta g_{0}$. Subject to certain assumptions, the first subleading term in (3.7) has been computed in [2].

For our later discussion of the spectrum, it will be useful to mention that in the short time limit $\chi \rightarrow 0$ we have

$$
\mathcal{F}(\chi)=\sum_{m=1}^{\infty} c_{m}^{2} \chi^{h_{m}}, \text { where, } c_{m}^{2}=\alpha_{0} \frac{\left(h_{m}-1 / 2\right)}{\pi \tan \left(\pi h_{m} / 2\right) k^{\prime}\left(h_{m}\right)} \frac{\Gamma\left(h_{m}\right)^{2}}{\Gamma\left(2 h_{m}\right)},
$$

\footnotetext{
${ }^{7}$ Since $b \sim g_{0}^{-1 / 2}$ it follows that $G^{\kappa} \sim g_{0}^{1 / 2}$. This dependence is unlike $G$ which scales as $G \sim g_{0}^{-1 / 2}$.

${ }^{8}$ From now on, we will use $K$ for $K^{\psi}, \mathcal{F}$ for $\mathcal{F}^{\psi}$ and $\mathcal{F}_{0}$ for $\mathcal{F}_{0}^{\psi}$.
} 


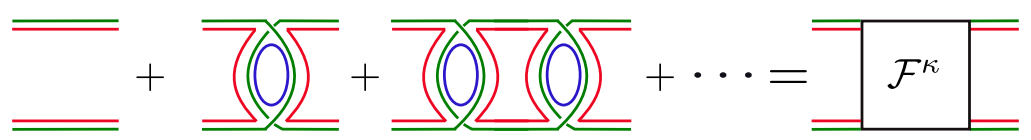

Figure 10. Diagrams contributing to 4 point function of $\kappa$.

where $\alpha_{0}=g_{0}^{2} b^{4}, b=\left(4 \pi g_{0}^{2}\right)^{-1 / 4}$ and $h_{m}$ is the $m^{t h}$ root $^{9}$ of the equation

$$
3 \tan \frac{\pi(h-1 / 2)}{2}=1-2 h .
$$

\section{$3.2 .2\langle\kappa \kappa \kappa \kappa\rangle$}

The gauge invariant four point function of the probe fields has the following structure:

$$
\frac{1}{N^{4}} \sum_{a_{1}, b_{1}, a_{2}, b_{2}}\left\langle\kappa_{a_{1} b_{1}}\left(t_{1}\right) \kappa_{a_{1} b_{1}}\left(t_{2}\right) \kappa_{a_{2} b_{2}}\left(t_{3}\right) \kappa_{a_{2} b_{2}}\left(t_{4}\right)\right\rangle=G^{\kappa}\left(t_{1}, t_{2}\right) G^{\kappa}\left(t_{3}, t_{4}\right)+\frac{1}{N^{2}} \mathcal{F}^{\kappa}\left(t_{1}, t_{2} ; t_{3}, t_{4}\right)
$$

and $\mathcal{F}^{\kappa}$ is given by sum of ladders shown in figure 10. The first connected piece of $\mathcal{F}^{\kappa}$ is obtained by cutting two $\kappa$ lines in the lower melon of figure 8 . Subsequent diagrams can be obtained by cutting more complicated melons although it is easier to think of these ladder diagrams as being obtained by successively adding rungs constructed only from $\psi$-fields. The sum of ladders in figure 10 has the same structure as $\mathcal{F}^{\psi}$ and one gets

$$
\mathcal{F}^{\kappa}=\frac{1}{1-K^{\kappa}} \mathcal{F}_{0}^{\kappa}=\frac{g_{0}^{2}}{g_{1}^{2}} \frac{1}{1-\frac{1}{3} K^{\psi}} \mathcal{F}_{0}^{\psi},
$$

where we have used

$$
\begin{aligned}
& K^{\kappa}\left(t_{1}, t_{2} ; t_{3}, t_{4}\right)=-g_{1}^{2} G^{\kappa}\left(t_{1}, t_{3}\right) G^{\kappa}\left(t_{2}, t_{4}\right) G^{\psi}\left(t_{3}, t_{4}\right)^{2}=\frac{1}{3} K^{\psi}\left(t_{1}, t_{2} ; t_{3}, t_{4}\right), \\
& \mathcal{F}_{0}^{\kappa}\left(t_{1}, t_{2} ; t_{3}, t_{4}\right)=-G^{\kappa}\left(t_{1}, t_{3}\right) G^{\kappa}\left(t_{2}, t_{4}\right)+G^{\kappa}\left(t_{1}, t_{4}\right) G^{\kappa}\left(t_{2}, t_{3}\right)=\frac{g_{0}^{2}}{g_{1}^{2}} \mathcal{F}_{0}^{\psi}\left(t_{1}, t_{2} ; t_{3}, t_{4}\right) .
\end{aligned}
$$

Similarly to (3.6) we define in the strict conformal limit

$$
\mathcal{F}^{\kappa}\left(t_{1}, t_{2} ; t_{3}, t_{4}\right)=G^{\kappa}\left(t_{12}\right) G^{\kappa}\left(t_{34}\right) \mathcal{F}^{\kappa}(\chi) .
$$

The computation of $\mathcal{F}^{\kappa}$ is similar to that of $\mathcal{F}^{\psi}$. However due to the extra factor of $1 / 3$ in (3.11), there are key differences, most significant one being that there are no divergences coming from $h=2$ subspace anymore. In fact there are no divergences at all, since the equation $1-\frac{1}{3} k_{c}(h)=0$ does not admit any solution of the form $h=2 n, n \in \mathbb{Z}$. Lack of divergence entails that $\mathrm{SL}(2, \mathbb{R})$ symmetry is exact for $\mathcal{F}^{\kappa}$ and consequently there is no new pseudo-Goldstone mode. This can be interpreted as the behaviour of probe fields to leading order not being affected by breaking of near horizon $A d S_{2}$ symmetry. This also results in Lyapunov exponent for $\mathcal{F}^{\kappa}$ being non-maximal.

\footnotetext{
${ }^{9}$ We define $h_{0}=2$.
} 

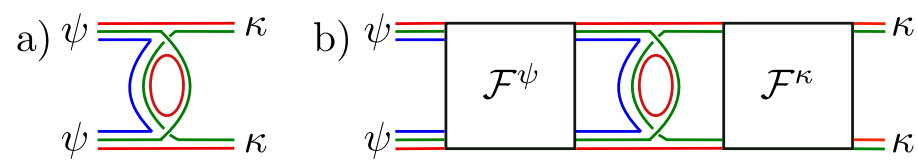

Figure 11. a) Basic vertex contributing to $\langle\psi \psi \kappa \kappa\rangle$. b) One can continue adding rungs on both side of the first diagram to obtain this structure at all orders.

Finally, in $\chi \rightarrow 0$ limit, we have

$$
\mathcal{F}^{\kappa}(\chi)=\sum_{m=1}^{\infty} \widetilde{c}_{m}^{2} \chi^{\widetilde{h}_{m}}, \text { where, } \widetilde{c}_{m}^{2}=9 \alpha_{0} \frac{\left(\widetilde{h}_{m}-1 / 2\right)}{\pi \tan \left(\pi \widetilde{h}_{m} / 2\right) k^{\prime}\left(\widetilde{h}_{m}\right)} \frac{\Gamma\left(\widetilde{h}_{m}\right)^{2}}{\Gamma\left(2 \widetilde{h}_{m}\right)},
$$

where $b$ and $\alpha_{0}$ are given below (3.8) and $\tilde{h}_{m}$ is $m^{t h}$ root of the equation

$$
\tan \left(\frac{\pi h}{2}-\frac{\pi}{4}\right)=1-2 h
$$

Note there is a natural correspondence between the conformal dimensions $\widetilde{h}_{m}$-s and $h_{m}$-s.

\subsection{3 $\langle\psi \psi \kappa \kappa\rangle$}

The mixed four point function is

$$
\frac{1}{N^{5}} \sum_{a_{1}, b_{1}, c_{1}, a_{2}, b_{2}}\left\langle\psi_{a_{1} b_{1} c_{1}}\left(t_{1}\right) \psi_{a_{1} b_{1} c_{1}}\left(t_{2}\right) \kappa_{a_{2} b_{2}}\left(t_{3}\right) \kappa_{a_{2} b_{2}}\left(t_{4}\right)\right\rangle=G^{\psi}\left(t_{1}, t_{2}\right) G^{\kappa}\left(t_{3}, t_{4}\right)+\frac{1}{N^{3}} \mathcal{F}^{\psi \kappa}
$$

The simplest contribution to $\mathcal{F}^{\psi \kappa}$ comes from a diagram obtained by cutting a $\psi$ line and a $\kappa$ line in the lower melon of figure 8. This is a ladder with a single rung as shown in figure 11a, where the uncontracted lines on the left correspond to $\psi$ fields and thus have three unresolved components while those on the right correspond to $\kappa$ fields and have two unresolved lines. We can express it as

$$
\frac{2 g_{1}}{3 g_{0}} \int d t d t^{\prime} K\left(t_{1}, t_{2} ; t, t^{\prime}\right) \mathcal{F}_{0}^{\kappa}\left(t, t^{\prime} ; t_{3}, t_{4}\right)=\frac{2 g_{1}}{3 g_{0}}\left(K * \mathcal{F}_{0}^{\kappa}\right)\left(t_{1}, t_{2} ; t_{3}, t_{4}\right) .
$$

Now to generate the set of graphs which contribute to $\mathcal{F}^{\psi \kappa}$ at leading order in the $1 / N$ expansion, one should add appropriate rungs on both sides of the given rung as presented in figure 11b. All the rungs on both the left and the right correspond to $\psi$-fields being exchanged as do all the side-rails except for two internal rails and the two uncontracted lines on the right, which represent $\kappa$-fields. The final result follows immediately from figure $11 \mathrm{~b}$

$$
\mathcal{F}^{\psi \kappa}=\frac{2 g_{0}}{3 g_{1}} \frac{K}{(1-K)\left(1-\frac{1}{3} K\right)} \mathcal{F}_{0}^{\psi} .
$$

Similarly as for $\mathcal{F}^{\psi}$ we have a component which depends only on the conformal cross ration $\chi$ and an additional component which breaks conformal symmetry:

$$
\frac{\mathcal{F}^{\psi \kappa}\left(t_{1}, t_{2}, t_{3}, t_{4}\right)}{G^{\kappa}\left(t_{12}\right) G\left(t_{34}\right)}=\mathcal{F}_{h \neq 2}^{\psi \kappa}(\chi)+\mathcal{F}_{h=2}^{\psi \kappa}\left(t_{1}, t_{2}, t_{3}, t_{4}\right) .
$$


The conformal part is evaluated by using same techniques used to evaluate $\mathcal{F}^{\psi}$ and $\mathcal{F}^{\kappa}$. In $\chi \rightarrow 0$ limit, one has

$$
\mathcal{F}^{\psi \kappa}=\sum_{m=1}^{\infty}\left[c_{m}^{2} \chi^{h_{m}}-\widetilde{c}_{m}^{2} \chi^{\widetilde{h}_{m}}\right]
$$

\subsection{Chaos}

In a chaotic quantum system, out of time order correlation functions grow exponentially. ${ }^{10}$ In the present case, there are three out of time order correlators that one may look at in order to diagnose chaos, namely

$$
\begin{aligned}
F^{\psi}\left(t_{1}, t_{2}\right) & =\operatorname{Tr}\left[y \psi_{a b c}\left(t_{1}\right) \psi_{a^{\prime} b^{\prime} c^{\prime}}(0) y \psi_{a b c}\left(t_{2}\right) \psi_{a^{\prime} b^{\prime} c^{\prime}}(0)\right] \\
F^{\kappa}\left(t_{1}, t_{2}\right) & =\operatorname{Tr}\left[y \kappa_{a b}\left(t_{1}\right) \kappa_{a^{\prime} b^{\prime}}(0) y \kappa_{a b}\left(t_{2}\right) \kappa_{a^{\prime} b^{\prime}}(0)\right] \\
F^{\psi \kappa}\left(t_{1}, t_{2}\right) & =\operatorname{Tr}\left[y \psi_{a b c}\left(t_{1}\right) \kappa_{a^{\prime} b^{\prime} c^{\prime}}(0) y \psi_{a b c}\left(t_{2}\right) \kappa_{a^{\prime} b^{\prime}}(0)\right]
\end{aligned}
$$

where, $y=\rho(\beta)^{1 / 4}, \rho(\beta)$ being the density matrix at inverse temperature $\beta$ and repeated indices are summed over.

For $F^{\psi}$, rungs are given by

$$
K_{R}\left(t_{1}, t_{2} ; t_{3}, t_{4}\right)=3 g_{0}^{2} G_{R}\left(t_{1}, t_{3}\right) G_{R}\left(t_{2}, t_{4}\right) G_{l r}\left(t_{3}, t_{4}\right)^{2}
$$

where $G_{R}$ is the retarded Green's function and $G_{l r}$ is a Wightman correlator:

$$
G_{R}(t)=2 b \cos (\pi \Delta) \theta(t)\left[\frac{\pi}{\beta \sinh \frac{\pi t}{\beta}}\right]^{2 \Delta}, \quad G_{l r}(t)=b\left[\frac{\pi}{\beta \sinh \frac{\pi t}{\beta}}\right]^{2 \Delta} .
$$

When $F^{\psi}$ is acted upon by $K_{R}$, one gets back $F^{\psi}$, except the $0^{\text {th }}$ piece. But this piece has a $G_{R}\left(t_{1}\right) G_{R}\left(t_{2}\right)$ in it, which is negligible for large $t_{1}, t_{2}$. So, in this limit, $F^{\psi}$ is an eigenfunction of $K_{R}$ with eigenvalue 1 . In order to solve for this, one can make an ansatz

$$
F^{\psi}\left(t_{1}, t_{2}\right)=e^{\lambda_{L}^{\psi}\left(t_{1}+t_{2}\right) / 2} f\left(t_{12}\right)
$$

$\lambda_{L}^{\psi}$ being the Lyapunov coefficient for $F^{\psi}$. Looking at eigenfunctions of $K_{R}$, one finds they are given by

$$
\ell_{h}\left(t_{1}, t_{2}\right)=\frac{e^{-h \frac{\pi}{\beta}\left(t_{1}+t_{2}\right)}}{\left[\cosh \frac{\pi}{\beta} t_{12}\right]^{1 / 2-h}}
$$

with eigenvalue

$$
k_{R}(h)=\frac{\Gamma(5 / 2) \Gamma(1 / 2-h)}{\Gamma(3 / 2) \Gamma(3 / 2-h)},
$$

\footnotetext{
${ }^{10}$ For the SYK model, this growth is followed by an exponential decay [5] and then a power law decay [35].
} 
in the conformal limit. It is then easy to see that only solution for $k_{R}(h)=1$ is $h=-1$. In large $t_{1}, t_{2}$, one has

$$
F^{\psi}\left(t_{1}, t_{2}\right)=\frac{e^{\pi\left(t_{1}+t_{2}\right) / \beta}}{\left[\cosh \frac{\pi}{\beta} t_{12}\right]^{1 / 2-h}}
$$

Comparing with (3.23), we see

$$
\lambda_{L}^{\psi}=2 \pi / \beta
$$

which is maximal Lyapunov coefficient [17] and black holes are known to saturate this bound.

Coming to $F^{\kappa}$, rungs are given by $K_{R}^{\kappa}=\frac{1}{3} K_{R}$. We make an ansatz similar to (3.23). Using same line of argument, we now look for the solution $k_{R}^{\kappa}(h)=1$, or $k_{R}(h)=3$. The solution to this equation is $h=0$, suggesting that $F^{\kappa}$ is non-chaotic to leading order in $1 / N$.

Finally we come to $F^{\psi \kappa}$. It has two kinds of ladders, one containing $K_{R}$ as rungs and another $\frac{1}{3} K_{R}$. The leading contribution to Lyapunov coefficient comes from $K_{R}$ rungs and using similar line of argument we have

$$
\lambda_{L}^{\psi \kappa}=2 \pi / \beta
$$

One might wonder if the situation for $F^{\kappa}$ can be improved by considering models with higher $q$. Such models can be obtained by starting with colored models (see section 4 ) and then uncoloring them. For large times, one has $F^{\kappa} \sim e^{-h \frac{\pi}{\beta}\left(t_{1}+t_{2}\right)}, h$ being the solution to the equation

$$
\frac{\Gamma(3-2 / q) \Gamma(2 / q-h)}{\Gamma(1+2 / q) \Gamma(2-2 / q-h)}=q-1
$$

It is easy to see that $h=0$ satisfies this equation. Thus to leading order in $1 / N, F^{\kappa}$ remains non-chaotic in such models.

Finally, coming to the question which four point functions are maximally chaotic, in A, we show if a four point function has any positive power of $(1-K)$ in the denominator, corresponding OTOC is maximally chaotic.

\subsection{Spectrum}

The SYK model (and its tensorial cousins) are widely believed to constitute an example of so called $N A d S_{2} / N C F T_{1}$ [2]. Conformal primaries of the SYK model correspond to bulk fields whose masses are determined by the scaling dimensions of the primaries. To compute these, one first expresses the gauge invariant fermion bilinear as (we write it for the KT model)

$$
\sum_{a, b, c} \psi_{a b c}\left(t_{1}\right) \psi_{a b c}\left(t_{2}\right)=N^{3 / 2} G\left(t_{12}\right) \sum_{n} c_{\psi, n}\left|t_{12}\right|^{h_{n}^{\prime}} \mathcal{O}_{\psi, n}\left(\frac{t_{1}+t_{2}}{2}\right),
$$


where $h_{n}^{\prime}$ is the conformal dimension of $n^{t h}$ primary, i.e.

$$
\left\langle\mathcal{O}_{\psi, m}\left(t_{1}\right) \mathcal{O}_{\psi, n}\left(t_{2}\right)\right\rangle=\frac{\delta_{m n}}{\left|t_{12}\right|^{2 h_{n}^{\prime}}} .
$$

. To compute $h_{n}^{\prime}$-s and $c_{\psi, n}$-s, one notes in the limit $t_{12} \rightarrow 0, t_{34} \rightarrow 0$,

$$
\begin{aligned}
& \sum_{\substack{a, b, c ; \\
a^{\prime}, b^{\prime}, c^{\prime}}}\left\langle\psi_{a b c}\left(t_{1}\right) \psi_{a b c}\left(t_{2}\right) \psi_{a^{\prime} b^{\prime} c^{\prime}}\left(t_{3}\right) \psi_{a^{\prime} b^{\prime} c^{\prime}}\left(t_{4}\right)\right\rangle \\
= & N^{3} G\left(t_{12}\right) G\left(t_{34}\right) \sum_{m, n} c_{\psi, m} c_{\psi, n}\left|t_{12}\right|^{h_{m}^{\prime}}\left|t_{34}\right|^{h_{n}^{\prime}}\left\langle\mathcal{O}_{\psi, m}\left(\frac{t_{1}+t_{2}}{2}\right) \mathcal{O}_{\psi, n}\left(\frac{t_{3}+t_{4}}{2}\right)\right\rangle \\
= & N^{3} G\left(t_{12}\right) G\left(t_{34}\right) \sum_{n} c_{\psi, n}^{2} \frac{\left|t_{12}\right|^{h_{n}^{\prime}}\left|t_{34}\right|^{h_{n}^{\prime}}}{\left|t_{13}\right|^{2 h_{n}^{\prime}}} .
\end{aligned}
$$

Again in the same limit, one has

$$
\sum_{\substack{a, b, c, c \\ a^{\prime}, b^{\prime}, c^{\prime}}}\left\langle\psi_{a b c}\left(t_{1}\right) \psi_{a b c}\left(t_{2}\right) \psi_{a^{\prime} b^{\prime} c^{\prime}}\left(t_{3}\right) \psi_{a^{\prime} b^{\prime} c^{\prime}}\left(t_{4}\right)\right\rangle=N^{3} G\left(t_{12}\right) G\left(t_{34}\right) \sum_{m=1}^{\infty} c_{m}^{2}\left(\frac{t_{12} t_{34}}{t_{13} t_{24}}\right)^{h_{m}}
$$

where $c_{m}^{2}$ are given in (3.8). Comparing (3.32) and (3.33), one has

$$
h_{\psi, n}^{\prime}=h_{n}, c_{\psi, n}=c_{n}
$$

In the dual bulk theory, corresponding to a boundary primary of dimension $h_{n}$ one gets a bulk field $\phi_{n}$ of mass $m_{n}^{2}=h_{n}\left(h_{n}-1\right)$.

In the present case, there are extra primaries due to $\kappa$-fields. We restrict to the simplest case of (3.1). Then similarly one gets

$$
\sum_{a, b} \kappa_{a b}\left(t_{1}\right) \kappa_{a b}\left(t_{2}\right)=N G^{\kappa}\left(t_{12}\right) \sum_{n} \widetilde{c}_{n}\left|t_{12}\right|^{\widetilde{h}_{n}} \mathcal{O}_{\kappa, n}\left(\frac{t_{1}+t_{2}}{2}\right) .
$$

These primaries lead to a new set of bulk fields $\phi_{n}^{\prime}$ with masses $\widetilde{m}_{n}^{2}=\widetilde{h}_{n}\left(\widetilde{h}_{n}-1\right)$.

Since $h_{m} \neq \widetilde{h}_{n} \forall m, n,(3.30)$ and (3.35) implies $\mathcal{F}^{\psi \chi}=0$. However direct computations show $\mathcal{F}^{\psi \chi}$ is non zero. This can be incorporated by following ansatz

$$
\begin{aligned}
\sum_{a, b, c} \psi_{a b c}\left(t_{1}\right) \psi_{a b c}\left(t_{2}\right)= & N^{3 / 2} G\left(t_{12}\right) \sum_{n} c_{n}\left|t_{12}\right|^{h_{n}} \mathcal{O}_{\psi, n}\left(\frac{t_{1}+t_{2}}{2}\right) \\
& -N G\left(t_{12}\right) \sum_{n} \widetilde{c}_{n}\left|t_{12}\right|^{\widetilde{h}_{n}} \mathcal{O}_{\kappa, n}\left(\frac{t_{1}+t_{2}}{2}\right), \\
\sum_{a, b} \kappa_{a b}\left(t_{1}\right) \kappa_{a b}\left(t_{2}\right)= & N G^{\kappa}\left(t_{12}\right) \sum_{n} \widetilde{c}_{n}\left|t_{12}\right|^{\widetilde{h}_{n}} \mathcal{O}_{\kappa, n}\left(\frac{t_{1}+t_{2}}{2}\right) \\
& +N^{1 / 2} G^{\kappa}\left(t_{12}\right) \sum_{n} c_{n}\left|t_{12}\right|^{h_{n}} \mathcal{O}_{\psi, n}\left(\frac{t_{1}+t_{2}}{2}\right) .
\end{aligned}
$$



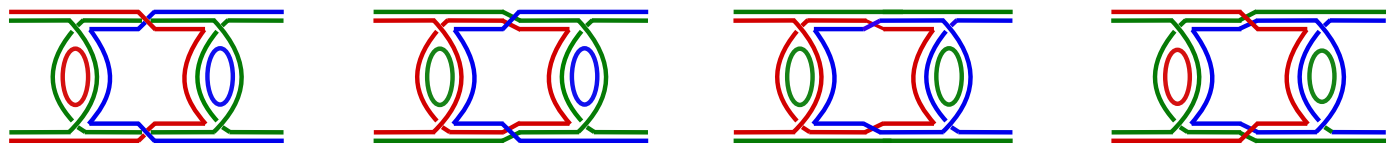

Figure 12. Simplest leading contributions to $\left\langle\kappa^{(12)} \kappa^{(12)} \kappa^{(23)} \kappa^{(23)}\right\rangle$.

\subsection{Adding additional fields}

In section 2.2, we considered a general class of models which are obtained by adding additional fields in KT model but remain solvable at large $N$ in the deep infrared. We then considered a particular case in section 2. Here we shed some light on the general case.

First we restore permutation symmetry between the three copies of $\operatorname{SO}(N)$. This amounts to introducing three new fields to the original KT model: $\kappa^{(12)}, \kappa^{(13)}, \kappa^{(23)}$. This is implemented by replacing the probe term in (3.1) by the following term

$$
V_{1}=\frac{g_{1}}{2 N^{3 / 2}}\left(\psi_{a_{1} b_{1} c_{1}} \psi_{a_{2} b_{2} c_{1}} \kappa_{a_{1} b_{2}}^{(12)} \kappa_{a_{2} b_{1}}^{(12)}+\psi_{a_{1} b_{1} c_{1}} \psi_{a_{2} b_{1} c_{2}} \kappa_{a_{2} c_{1}}^{(13)} \kappa_{a_{1} c_{2}}^{(13)}+\psi_{a_{1} b_{1} c_{1}} \psi_{a_{1} b_{2} c_{2}} \kappa_{b_{1} c_{2}}^{(23)} \kappa_{b_{2} c_{1}}^{(23)}\right) .
$$

It is easy to check that $G^{\kappa^{12}}=G^{\kappa^{13}}=G^{\kappa^{23}}=G^{\kappa}$, where $G^{\kappa}$ is same as (3.3).

Coming to four point functions, again it is easy to check that $\left\langle\psi \psi \kappa^{(i j)} \kappa^{(i j)}\right\rangle$ is the same for all three $(i j)$-s and has the same expression as in (3.15). Similarly the four point function $\left\langle\kappa^{(i j)} \kappa^{(i j)} \kappa^{(i j)} \kappa^{(i j)}\right\rangle$ is also same for all three $(i j)$-s and same as (3.10). Along with these, now there is a new four point function $\left\langle\kappa^{(i j)} \kappa^{(i j)} \kappa^{(j k)} \kappa^{(j k)}\right\rangle$. This is the same for all three choices of $(i j),(j k)$ by permutation symmetry. As an example, we consider the case $(i j)=(12),(j k)=(23)$. It has the following structure

$$
\frac{1}{N^{4}} \sum_{a, b, b^{\prime}, c^{\prime}}\left\langle\kappa_{a b}^{(12)}\left(t_{1}\right) \kappa_{a b}^{(12)}\left(t_{2}\right) \kappa_{b^{\prime} c^{\prime}}^{(23)}\left(t_{3}\right) \kappa_{b^{\prime} c^{\prime}}^{(23)}\left(t_{4}\right)\right\rangle=G^{\kappa}\left(t_{12}\right) G^{\kappa}\left(t_{34}\right)+\frac{1}{N^{3}} \widetilde{\mathcal{F}}^{\kappa},
$$

and the simplest leading contributions to $\widetilde{\mathcal{F}}^{\kappa}$ are shown in figure 12. All four diagrams contribute the same, which then add up to give

$$
\widetilde{\mathcal{F}}^{\kappa}=\frac{64 g_{0}^{2}}{9 g_{1}^{2}} \frac{\left(K^{\psi}\right)^{2}}{\left(1-K^{\psi}\right)\left(1-\frac{1}{3} K^{\psi}\right)^{2}} \mathcal{F}_{0}^{\psi} .
$$

$\widetilde{\mathcal{F}}$ can be expressed as a sum of a conformal and a conformal symmetry breaking part.

$$
\frac{\widetilde{\mathcal{F}}^{\kappa}\left(t_{1}, t_{2} ; t_{3}, t_{4}\right)}{G^{\kappa}\left(t_{12}\right) G^{\kappa}\left(t_{34}\right)}=\widetilde{\mathcal{F}}_{h \neq 2}(\chi)+\widetilde{\mathcal{F}}_{h=2}\left(t_{1}, t_{2} ; t_{3}, t_{4}\right) .
$$

For small $\chi$ the conformal part reduces to

$$
\widetilde{\mathcal{F}}=\sum_{m=1}^{\infty}\left[16 c_{m}^{2} \chi^{h_{m}}+\frac{64}{3} \widetilde{c}_{m}^{2} \chi^{\widetilde{h}_{m}}\right]
$$

We do not attempt to evaluate the non-conformal part here. But we note in passing that it scales as $\beta g_{0}$, same as the corresponding piece of $\psi^{4}$ and $\psi^{2} \kappa^{2}$ four point functions. 

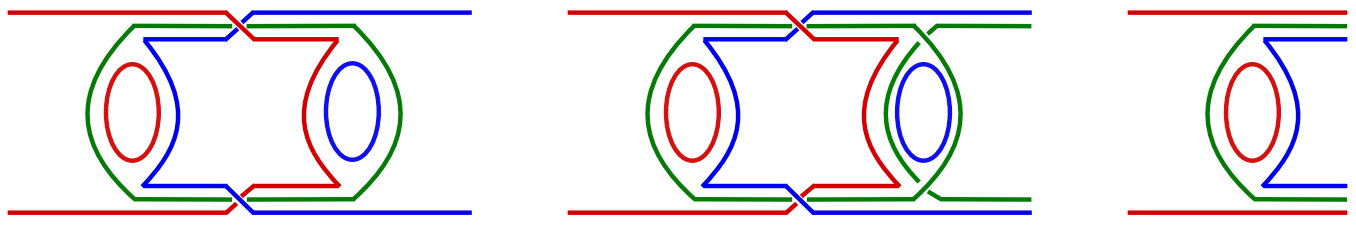

Figure 13. Simplest leading contributions to four point functions in (3.45).

Generalizing the chaos computations of section 3.3, it follows that the corresponding OTOC

$$
\widetilde{F}^{\kappa}\left(t_{1}, t_{2}\right)=\operatorname{Tr}\left[y \kappa^{(i j)}\left(t_{1}\right) y \kappa^{(i j)}(0) y \kappa^{(i j)}\left(t_{2}\right) y \kappa^{(i j)}(0)\right]
$$

also has maximal Lyapunov coefficient $\lambda_{L}=2 \pi / \beta$.

Next, one can add fields carrying a single index. This corresponds to introducing the following interaction in the Hamiltonian:

$$
V_{2}=\frac{g_{2}}{N^{3 / 2}}\left(\psi_{a_{1} b_{1} c_{1}} \kappa_{b_{2} c_{1}}^{(23)} \kappa_{a_{1} b_{2}}^{(12)} \eta_{b_{1}}^{(2)}+\psi_{a_{1} b_{1} c_{1}} \kappa_{a_{1} c_{2}}^{(13)} \kappa_{b_{1} c_{2}}^{(23)} \eta_{c_{1}}^{(3)}+\psi_{a_{1} b_{1} c_{1}} \kappa_{a_{2} b_{1}}^{(12)} \kappa_{a_{2} c_{1}}^{(13)} \eta_{a_{1}}^{(1)}\right)
$$

Starting with propagators, first we note all three $G^{\eta_{i}}(i=1,2,3)$ are the same by permutation symmetry and we will call them $G^{\eta}$. The Schwinger Dyson equation for $G^{\eta}$ turns out to be very similar to $G^{\psi}$ and $G^{\kappa}$ and has the following solution

$$
G^{\eta}(t)=\frac{g_{1}^{2}}{g_{2}^{2}} G(t)=\frac{b g_{2}^{2}}{g_{1}^{2}} \frac{\operatorname{sgn}(t)}{|t|^{1 / 2}} .
$$

The four point functions involving only $\psi$ and $\kappa$ are not affected by $V_{2}$ to leading order. There are quite a few new four point functions involving $\eta$. Among these, the following four point functions break conformal symmetry:

$$
\left\langle\eta^{i} \eta^{i} \eta^{j} \eta^{j}\right\rangle,\left\langle\eta^{i} \eta^{i} \kappa^{(j k)} \kappa^{(j k)}\right\rangle,\left\langle\eta^{i} \eta^{i} \psi \psi\right\rangle \quad i \neq j \neq k
$$

where sum over suppressed indices are understood. Leading conformal symmetry breaking contributions to these four points functions can be obtained by rungs added with the diagrams in figure 13. Some of these four point functions have conformal leading contributions from other diagrams as well. Although we do not evaluate these four point functions, we note that the conformal symmetry breaking part scales as $\beta g_{0}$ for all of them and the conformal spectrum contains fields of scaling dimensions $h_{m}$ and $\widetilde{h}_{m}$ only. The breaking of conformal symmetry follows form presence of a $\psi$ rail in the ladder diagrams. The scaling of conformal symmetry breaking part follows from the fact that there is only one such rail for each of them. The scaling dimensions follow from the fact that these four point functions have poles at same values of $K$ as those involving only $\kappa$ and $\psi$ fields. OTOC-s corresponding to these four point functions are maximally chaotic.

Other four point functions involving $\eta$ do not break conformal symmetry to leading order in $1 / N$. Consequently corresponding OTOC-s do not exhibit maximal chaos. 


\section{Colored probe model}

We first briefly recall the Gurau-Witten model [18]. For $D+1$ colors, the model contains $D+1$ Majorana fermions $\psi^{0}, \psi^{1}, \ldots, \psi^{D}$ and one associates a global $\mathrm{SO}(N)$ symmetry to each pair $(i j)$ : a fermion $\psi^{i}$ sits in fundamental of $\operatorname{SO}(N)_{i j} \forall j \neq i$. Thus each fermion carries $D$ indices and each index runs from 1 to $N$. For example, writing all the indices of $\psi^{0}$, one would have $\psi_{i_{1} i_{2} \ldots i_{D}}^{0}$ and so on. The Hamiltonian for this model is given by

$$
H_{G W}=\frac{g_{0}}{N^{D(D+1) / 4}} \psi^{0} \psi^{1} \ldots \psi^{D},
$$

where we have suppressed the indices. At leading order in $1 / N$ this model leads to same physics as SYK model. Further it had the advantage over SYK model of being fully quantum mechanical. One can further "uncolor" [32] this model to get the KT model [21].

Now we add probe fields to this model. We would denote a probe field, obtained from say $\psi^{0}$ by removing the index $i_{01}$, as $\psi^{0 \widehat{1}}$. Similarly $\psi^{2 \widehat{0} \widehat{1}}$ denotes a field obtained from $\psi^{2}$ by removing indices $i_{02}, i_{12}$. Since we have already gathered some understanding of probe fields in section 3, we add all possible probe fields at one go. This amounts to adding the following interactions with (4.1):

$$
\begin{aligned}
V_{1} & =\frac{g_{1}}{2 !(D-1) ! N^{D(D+1) / 4}} \epsilon_{c_{0} \ldots c_{D}} \psi^{c_{0} \widehat{c_{1}}} \psi^{c_{1} \widehat{c_{0}}} \psi^{c_{2}} \ldots \psi^{c_{D}}, \\
V_{2} & =\frac{g_{2}}{2 !(D-2) ! n^{D(D+1) / 4}} \epsilon_{c_{0} \ldots c_{D}} \psi^{c_{0} \widehat{c_{1}} \widehat{c_{2}}} \psi^{c_{1} \widehat{c_{0}}} \psi^{c_{2} \widehat{c_{0}}} \psi^{c_{3}} \ldots \psi^{c_{D}}, \\
& \vdots \\
V_{p} & =\frac{g_{p}}{p !(D-p) ! n^{D(D+1) / 4}} \epsilon_{c_{0} \ldots c_{D}} \psi^{c_{0} \widehat{c_{1}} \ldots \widehat{c_{p}}} \psi^{c_{1} \widehat{c_{0}}} \ldots \psi^{c_{p} \widehat{c_{0}}} \psi^{c_{p+1}} \ldots \psi^{c_{D}}, \\
& \vdots \\
V_{D-1} & =\frac{g_{D-1}}{(D-1) ! n^{D(D+1) / 4}} \epsilon_{c_{0} \ldots c_{D}} \psi^{c_{0} \widehat{c_{1}} \ldots \widehat{c_{D}-1}} \psi^{c_{1} \widehat{c_{0}}} \ldots \psi^{c_{D-1} \widehat{c_{0}}} \psi^{c_{D}},
\end{aligned}
$$

where $c_{1}, \ldots, c_{D}$ run from 0 to $D$. The numerical prefactors are chosen to ensure that every term appears only once. E.g. for $D=3$, one has

$$
\begin{aligned}
V_{1}=\frac{g_{1}}{N^{3 / 2}}[ & \psi^{0, \widehat{1}} \psi^{1, \widehat{0}} \psi^{2} \psi^{3}+\psi^{0, \widehat{2}} \psi^{1} \psi^{2, \widehat{0}} \psi^{3}+\psi^{0, \widehat{3}} \psi^{1} \psi^{2} \psi^{3 \widehat{0}}+\psi^{0} \psi^{1 \widehat{2}} \psi^{2 \widehat{1}} \psi^{3} \\
& \left.+\psi^{0} \psi^{1 \widehat{3}} \psi^{2} \psi^{3 \widehat{1}}+\psi^{0} \psi^{1} \psi^{2 \widehat{3}} \psi^{3 \widehat{2}}\right] \\
V_{2}=\frac{g_{2}}{N^{3 / 2}}[ & \psi^{0 \widehat{10} \widehat{2}} \psi^{1 \hat{0}} \psi^{2 \widehat{0}} \psi^{3}+\psi^{0 \widehat{23}} \psi^{1} \psi^{2 \widehat{0}} \psi^{3 \widehat{0}}+\psi^{0 \widehat{13}} \psi^{1 \widehat{0}} \psi^{2} \psi^{3 \widehat{0}}+\psi^{0 \widehat{1}} \psi^{1 \widehat{03}} \psi^{2} \psi^{3 \widehat{1}} \\
& +\psi^{0 \widehat{1}} \psi^{1 \widehat{2} \widehat{0}} \psi^{2 \widehat{1}} \psi^{3}+\psi^{0} \psi^{1 \widehat{3} \widehat{2}} \psi^{2 \widehat{1}} \psi^{3 \widehat{1}}+\psi^{0 \widehat{2}} \psi^{1 \widehat{2}} \psi^{2 \widehat{0} \widehat{1}} \psi^{3}+\psi^{0 \widehat{2}} \psi^{1} \psi^{2 \widehat{30}} \psi^{3 \widehat{2}} \\
& \left.+\psi^{0} \psi^{1 \widehat{2}} \psi^{2 \widehat{1} \widehat{3}} \psi^{3 \widehat{2}}+\psi^{0 \widehat{3}} \psi^{1} \psi^{2 \widehat{3}} \psi^{3 \widehat{2} \widehat{0}}+\psi^{0 \widehat{3}} \psi^{1 \widehat{3}} \psi^{2} \psi^{3 \widehat{10}}+\psi^{0} \psi^{1 \widehat{3}} \psi^{2 \widehat{3}} \psi^{3 \widehat{1} \widehat{2}}\right],
\end{aligned}
$$

and so on. The analysis of four point functions is very similar to those of uncolored probe model and exhibit same pattern of conformal symmetry breaking as well as maximal chaos for a host of four point functions. 


\section{Discussion and future directions}

In this paper we have presented various tensor models which couple tensors of rank one and two to the tensor models of [18-20]. We have argued that these describe the interaction of probes with a near extremal black hole, in particular these models exhibit maximal chaos for a host of four point functions. All conformal symmetry breaking correlation functions are found to scale as $\beta g_{0}$. An obvious next step towards understanding this system better would be to study the validity of Eigenstate Thermalization Hypothesis (ETH) in this model and we intend to investigate this in a future work.

\section{Acknowledgments}

SM would like to thank Subhroneel Chakrabarti and Ashoke Sen for discussions. We would like to thank the anonymous referee for pointing out a numerical error in the first version of this article. This work was conducted within the ILP LABEX (ANR-10-LABX63) supported by French state funds managed by the ANR within the Investissements d'Avenir program (ANR-11-IDEX-0004-02) and supported partly by the CEFIPRA grant 5204-4. S.M. would like to thank Harish-Chandra Research Institite for hospitality, where part of the work was done.

\section{A Four point functions with maximal chaos}

For any four point function with form

$$
\mathcal{F}_{m, n,\left\{n_{i}\right\}}=\frac{K^{m}}{(1-K)^{n} \prod_{i}\left(1-\alpha_{i} K\right)^{n_{i}}} \mathcal{F}_{0},
$$

with $\alpha_{i}<1$ and $m, n, n_{i}$ positive integers, the corresponding OTOC $F_{m, n,\left\{n_{i}\right\}}\left(t_{1}, t_{2}\right)$ will have maximal Lyapunov coefficient. We note that if we act $F_{m, n,\left\{n_{i}\right\}}$ by $K_{m, n,\left\{n_{i}\right\}}^{R} \equiv$ $1-\left(1-K_{R}\right)^{n} \prod_{i}\left(1-\alpha_{i} K\right)^{n_{i}} / K_{R}^{m}$, we get back $F_{m, n,\left\{n_{i}\right\}}$ except for a piece that can be ignored in large $t_{1}, t_{2}$ limit. Thus in this limit $F_{m, n,\left\{n_{i}\right\}}\left(t_{1}, t_{2}\right)$ is an eigenfunction of the operator $K_{m, n,\left\{n_{i}\right\}}^{R}$ with eigenvalue 1 . Now the operator $K_{m, n,\left\{n_{i}\right\}}^{R}$ clearly commutes with $K_{R}$ and thus has same eigenfunctions, labelled by $h$ with eigenvalues given by

$$
k_{m, n,\left\{n_{i}\right\}}(h)=1-\left(1-k_{R}(h)\right)^{n} \prod_{i}\left(1-\alpha_{i} k_{R}(h)\right)^{n_{i}} / k_{R}(h)^{m} .
$$

It is clear that $k_{m, n,\left\{n_{i}\right\}}(h)=1 \Rightarrow k_{R}(h)=1$. We have previously mentioned (see 3.3) that $k_{R}(h)=1$ is satisfied for $h=-1$ with the eigenfunction $\ell_{-1}\left(t_{1}, t_{2}\right)$ given in (3.24). This essentially implies that in long time limit $F_{m, n,\left\{n_{i}\right\}}=\ell_{-1}$ and therefore has maximal Lyapunov coefficient $\lambda_{L}=2 \pi / \beta$.

$k_{m, n,\left\{n_{i}\right\}}=1$ is also solved by $k_{R}(h)=\alpha_{i}^{-1}$. But such solutions lead to sub-maximal contribution to Lyapunov coefficient, and therefore do not determine the large time growth of the corresponding OTOC. 
Open Access. This article is distributed under the terms of the Creative Commons Attribution License (CC-BY 4.0), which permits any use, distribution and reproduction in any medium, provided the original author(s) and source are credited.

\section{References}

[1] A. Kitaev, KITP strings seminar and Entanglement 2015 program, http://online.kitp.ucsb.edu/online/entangled15/.

[2] J. Maldacena and D. Stanford, Remarks on the Sachdev-Ye-Kitaev model, Phys. Rev. D 94 (2016) 106002 [arXiv: 1604.07818] [INSPIRE].

[3] K. Jensen, Chaos in AdS $S_{2}$ Holography, Phys. Rev. Lett. 117 (2016) 111601 [arXiv: 1605.06098] [INSPIRE].

[4] J. Engelsöy, T.G. Mertens and H. Verlinde, An investigation of $A d S_{2}$ backreaction and holography, JHEP 07 (2016) 139 [arXiv:1606.03438] [INSPIRE].

[5] J. Maldacena, D. Stanford and Z. Yang, Conformal symmetry and its breaking in two dimensional Nearly Anti-de-Sitter space, PTEP 2016 (2016) 12C104 [arXiv:1606.01857] [INSPIRE].

[6] A.M. García-García and J.J.M. Verbaarschot, Analytical Spectral Density of the Sachdev-Ye-Kitaev Model at finite N, Phys. Rev. D 96 (2017) 066012 [arXiv:1701.06593] [INSPIRE].

[7] J. Sonner and M. Vielma, Eigenstate thermalization in the Sachdev-Ye-Kitaev model, JHEP 11 (2017) 149 [arXiv:1707.08013] [INSPIRE].

[8] W. Fu, D. Gaiotto, J. Maldacena and S. Sachdev, Supersymmetric Sachdev-Ye-Kitaev models, Phys. Rev. D 95 (2017) 026009 [Addendum ibid. D 95 (2017) 069904] [arXiv: 1610.08917] [INSPIRE].

[9] N. Hunter-Jones, J. Liu and Y. Zhou, On thermalization in the SYK and supersymmetric SYK models, JHEP 02 (2018) 142 [arXiv: 1710.03012] [INSPIRE].

[10] M. Berkooz, P. Narayan, M. Rozali and J. Simón, Higher Dimensional Generalizations of the SYK Model, JHEP 01 (2017) 138 [arXiv:1610.02422] [INSPIRE].

[11] G. Turiaci and H. Verlinde, Towards a 2d QFT Analog of the SYK Model, JHEP 10 (2017) 167 [arXiv: 1701.00528] [INSPIRE].

[12] D.J. Gross and V. Rosenhaus, A Generalization of Sachdev-Ye-Kitaev, JHEP 02 (2017) 093 [arXiv: 1610.01569] [INSPIRE].

[13] J. Polchinski and V. Rosenhaus, The Spectrum in the Sachdev-Ye-Kitaev Model, JHEP 04 (2016) 001 [arXiv : 1601.06768] [INSPIRE].

[14] D.J. Gross and V. Rosenhaus, The Bulk Dual of SYK: Cubic Couplings, JHEP 05 (2017) 092 [arXiv: 1702.08016] [INSPIRE].

[15] D.J. Gross and V. Rosenhaus, All point correlation functions in SYK, JHEP 12 (2017) 148 [arXiv: 1710.08113] [INSPIRE].

[16] S.R. Das, A. Jevicki and K. Suzuki, Three Dimensional View of the SYK/AdS Duality, JHEP 09 (2017) 017 [arXiv: 1704.07208] [INSPIRE]. 
[17] J. Maldacena, S.H. Shenker and D. Stanford, A bound on chaos, JHEP 08 (2016) 106 [arXiv: 1503.01409] [INSPIRE].

[18] E. Witten, An SYK-Like Model Without Disorder, arXiv:1610.09758 [INSPIRE].

[19] R. Gurau, The 1/N expansion of colored tensor models, Annales Henri Poincaré 12 (2011) 829 [arXiv: 1011.2726] [INSPIRE].

[20] R. Gurau and V. Rivasseau, The 1/N expansion of colored tensor models in arbitrary dimension, EPL 95 (2011) 50004 [arXiv:1101.4182] [INSPIRE].

[21] I.R. Klebanov and G. Tarnopolsky, Uncolored random tensors, melon diagrams and the Sachdev-Ye-Kitaev models, Phys. Rev. D 95 (2017) 046004 [arXiv:1611.08915] [InSPIRE].

[22] C. Peng, M. Spradlin and A. Volovich, A Supersymmetric SYK-like Tensor Model, JHEP 05 (2017) 062 [arXiv:1612.03851] [INSPIRE].

[23] C. Krishnan, S. Sanyal and P.N. Bala Subramanian, Quantum Chaos and Holographic Tensor Models, JHEP 03 (2017) 056 [arXiv: 1612.06330] [INSPIRE].

[24] S. Choudhury, A. Dey, I. Halder, L. Janagal, S. Minwalla and R. Poojary, Notes on Melonic $O(N)^{q-1}$ Tensor Models, JHEP 06 (2018) 094 [arXiv:1707.09352] [INSPIRE].

[25] K. Bulycheva, I.R. Klebanov, A. Milekhin and G. Tarnopolsky, Spectra of Operators in Large N Tensor Models, Phys. Rev. D 97 (2018) 026016 [arXiv: 1707.09347] [InSPIRE].

[26] C. Peng, Vector models and generalized SYK models, JHEP 05 (2017) 129 [arXiv: 1704.04223] [INSPIRE].

[27] J. Yoon, SYK Models and SYK-like Tensor Models with Global Symmetry, JHEP 10 (2017) 183 [arXiv:1707.01740] [INSPIRE].

[28] N. Iizuka and J. Polchinski, A Matrix Model for Black Hole Thermalization, JHEP 10 (2008) 028 [arXiv: 0801.3657] [INSPIRE].

[29] N. Iizuka, T. Okuda and J. Polchinski, Matrix Models for the Black Hole Information Paradox, JHEP 02 (2010) 073 [arXiv:0808.0530] [INSPIRE].

[30] B. Michel, J. Polchinski, V. Rosenhaus and S.J. Suh, Four-point function in the IOP matrix model, JHEP 05 (2016) 048 [arXiv: 1602.06422] [INSPIRE].

[31] J. de Boer, E. Llabrés, J.F. Pedraza and D. Vegh, Chaotic strings in AdS/CFT, Phys. Rev. Lett. 120 (2018) 201604 [arXiv: 1709.01052] [INSPIRE].

[32] V. Bonzom, R. Gurau and V. Rivasseau, Random tensor models in the large $N$ limit: Uncoloring the colored tensor models, Phys. Rev. D 85 (2012) 084037 [arXiv:1202.3637] [INSPIRE].

[33] S. Dartois, V. Rivasseau and A. Tanasa, The $1 / N$ expansion of multi-orientable random tensor models, Annales Henri Poincaré 15 (2014) 965 [arXiv:1301.1535] [INSPIRE].

[34] A. Tanasa, The Multi-Orientable Random Tensor Model, a Review, SIGMA 12 (2016) 056 [arXiv: 1512.02087] [INSPIRE].

[35] D. Bagrets, A. Altland and A. Kamenev, Power-law out of time order correlation functions in the SYK model, Nucl. Phys. B 921 (2017) 727 [arXiv:1702.08902] [InSPIRE].

[36] S. Dartois, H. Erbin and S. Mondal, Conformality of $1 / N$ corrections in SYK-like models, arXiv: 1706.00412 [INSPIRE]. 